J. Sustain. Wireless Syst.

Vol.01/ No. 02

Pages: 103-115

http://irojournals.com/irosws/

DOI: https://doi.org/10.36548/jsws.2019.2.003

\title{
PERFORMANCE ANALYSIS AND QOS FRAMEWORK OF FLY WIIRELESS NETWORK
}

\author{
Dr. M. Durai Pandian, \\ Department of CSE, Vivekanandha College of Technology for Women, India. \\ Email id: svsduraipandian@gmail.com
}

\begin{abstract}
The spread out of wireless mesh network has made possible the extended range of communication network that are impractical due to environmental changes in a wired access point, these wireless mesh network does not require much competence to set it up as it can be set very fast at a cheap rate, and the conveyancing of messages in it happens by selecting the shortest path, these wireless mesh built-in with irrepressible and invulnerable identities come with an endurance to temporary congestion and individual node failure. This results in an architecture providing a better coverage, flaw indulgent with higher bandwidth compared to other wireless distributed systems. But faces the limitation on power conservation. The battery activated mesh nodes loses their resources on perception, processing and transmission of the data's, though these batteries or accumulators comes with energy regaining capability still draw backs show up as their nature of energy regaining are unexposed. So the performance analysis of fly wireless network which proposes a uninterrupted wireless mesh networks aims at providing a best measure of performance that is the best quality of service on the meshwork by providing an improved energy gleaning using potency segregation (IGPS) which empowers each node to have self- contained accumulation of energy achieving heightened adaption with energy consumption kept at a minimum. The gross functioning of the proposed is examined on the bases of delay and packet loss to prove the quality of service acquired.
\end{abstract}

Keywords: Wireless Mesh Network, Energy Gleaning, Power Conservation, Delay, Packet Loss, Measure Of Performance.

\section{Introduction}

In a world that strives to be connected without any seams, the growing demands are explosive and every minute counts to be productive. The continuous enhancements in the wireless network, acts as a powerful tool in boosting each minute to be productive by information sharing. The information sharing that is achieved using unconnected access to any of the network or application or documents. This allows a person to acquire the required information to the place where he is, without any interruptions. As the name suggests wireless network are unwired connection put into act and regulated using a radio waves. They are redistributed network helpful in all possible state of telecommunication and helps to retrieve three different details on generation of information they are local, neighbor, direct information. The wireless mesh network is a type of wireless network which is constructed using radio nodes that are structured in mesh topology. Where each node sends the information for the sake of the other and routing is performed by each node as each node connects directly without any controlling devices in between them to control their connection. This connectivity holds to the concepts behind ADHOC network. All the nodes in this mesh network are responsible for sending and receiving messages without any mediators as the nodes themselves plays 
J. Sustain. Wireless Syst.

Vol.01/ No. 02

Pages: 103-115

http://irojournals.com/irosws/

DOI: $\underline{\text { https://doi.org/10.36548/jsws.2019.2.003 }}$

the dual role of either taking forth the information or spreading them. These mesh nodes are radio transmitters of meagre size and performs the role of a wireless router which is the combination of access point and the router.it uses standard industrial protocol supported by the control and information protocol to communicate. Communication starts by one wired node sharing its connection with all the other nodes in its domain or neighborhood. The nodes further share the connection received to its other neighboring nodes forming a wireless covey of connectivity. These mesh mechanisms are remarkably beneficial over the regular wireless distribution system as they are irrepressible, invulnerable and can reshape on its own and shows better efficiency by achieving high throughput, simplicity is one of its main advantages. They also cover a quite large area by sending packets of data to just other node and allows the substitution of pathway by providing a rerouting between the alive unaffected nodes when one mesh node in network fails, thus providing an unaffected data transfer, the wireless mesh networks allots a stalwart frequency bands to intra-node information transfer and allows simultaneous data transfer from different contrivances withstanding the traffic. The infrastructure of the mesh network allows any development or changes to be done in a network without causing disturbance to the other points in the network. The nodes in the wireless mesh network are battery powered and the effectual transmission in these networks is performed by selecting path of nodes with higher battery ability, where the selection of nodes with low battery power would result in affecting the life span of the network and replenishment of battery would result in the degradation on the functioning of the system even though they are implemented with energy gleaner batteries. So it becomes necessary to have a battery in built with an improved energy gleaning so as to have an unaffected transmission without a degraded performance.

The wireless mesh networks which are capable of configuring, organizing, healing on its own can be dispersed anywhere any time, the important entrancing in wireless mesh networks would be the preservation of power. Since these wireless mesh network covers a wide area, uninterrupted power supply would be the main convention for data transmission, reorganizing, reconfiguring and healing nature of this wireless mesh network, apart from this the perception and processing nature of the nodes also acquires power. So an unbroken supply of power means to be a necessary demand for the better transmission. Though these nodes come up with batteries with inbuilt harvesting, there seems to be a drawback as the essential characteristics of these batteries are remain unexposed. The measure to overcome the drawback lead to the performance analysis of fly wireless network which proposes an improved energy gleaning methodology to be inbuilt into to the nodes to avoid the set downs caused during transmission.

This paper proposes an improved energy gleaning using potency segregation to improvise the battery power of the nodes achieving an interruption free transmission thus increasing the count of alive node encouraging transmission from different gadgets simultaneously allowing the power conservation of nodes to have a consummate quality of service in the gadget. The paper provides a performance check of the proposed based on the delay, total information delivered and reliability to measure the quality of service proffered by the proposed. 
J. Sustain. Wireless Syst.

Vol.01/ No. 02

Pages: 103-115

http://irojournals.com/irosws/

DOI: $\underline{\text { https://doi.org/10.36548/jsws.2019.2.003 }}$

The rest of the paper is organized as related works in section 2, proposed in section 3, measurement of quality of service in section 4 and conclusion in 5 .

\section{Related works}

Wang et.al [1] this is a converse about the various issues faced in the WMN wireless mesh network work based on multi beam antenna and also provides a deep converse on how medium access protocol and multi beam antenna can be developed. Bashir et.al [2].provides a detailed study on the movement of networks their management to have an improved energy efficiency in multi- UAV-adhoc networks Jianfeng et.al [3] this is an evaluation on a newly developed mac protocol to improvise the efficiency on communication by avoiding the majority of troubles caused due to synchronization of the beam and overlapping blocking problems in movement and reception. Teng et.al [4] provides with a better routing measurements to avail the sharing of networks efficiently between the base stations. It uses the packet level simulation to measure its performance and details about its energy efficiency in the multi-hop wireless network. Zhou et.al [5] uses integer nonlinear programming and optimized shortest routing path to achieve energy consumption in wireless mesh networks. Puniran et.al [6] uses a decode and forward method modeled by splitting up the power relays and switching relays offering a single relay usage for multiple origins to have an efficient energy harvesting module. Its performance are measured in terms of throughput. Attia et.al [7] achieves energy efficiency and to have an improved throughput it uses a clubbing of swarm and colony routing optimization. Li et.al [8] uses cluster based routing on wireless mesh networks to have better quality of service and reduce delay while transmitting information using wireless mesh network. Tu, Wanqing et.al [9] it is study based on how efficiently a communication can be managed using the designing of data driven QOS and QOE models. Singh et.al [10] shows that usage of wireless mesh network IEEE.802.11s helps to behave simple and be cost effective by introducing a different level of nodes called the mesh portals. This supports the wireless local area network mesh services and extends the transmission range by allowing the other nodes to forward the packets. Ertürk et.al [11] builds a strong wireless structure and measures performance metrics base on time slips and delay in the network. $\mathrm{Li}$, Shu et.al [12] uses a high level QOS possessed wireless mesh networks that proves to be energy efficient and it performance is compared to many other previous method to prove its efficiency.

\section{Proposed Work}

The pre-eminence evaluation of a particular data processing point depends on its type, observed characteristics, distances traversed, number of traversed hops and traffic loads. To start with pre-eminence the performance analysis of fly wireless network for wireless mesh networks initially starts with the conveyancing of the networks. The data 
J. Sustain. Wireless Syst.

Vol.01/ No. 02

Pages: 103-115

http://irojournals.com/irosws/

DOI: $\underline{\text { https://doi.org/10.36548/jsws.2019.2.003 }}$

processing points of the network are grouped in equal numbers together into a gauze structure in shape of a quad and the data processing points placed in the center of the gauze is elected to be its head and each head is limited with a specified amount of data. The grouping of the data processing units are given by equation (1)

$\mathrm{Sn}=\mathrm{Dp} / \mathrm{GSn}$

Where Sn represent the number of the groupings formed and Dp refers to the number of data processing point present and the GSn refers to the number of gauze structures available. The grouping formed on the center of the gauze attains the position of head this is done so that the all the data processing points available in the group would possess an equal energy. The information from each group head is collected using optimal scheduler based on its Pre-eminence and information from the data processing units in each group is collected using a contention protocol orderly so as to provide a proper simultaneous transmission to provide with good one way delay on speedy networks.

\section{Transmission through Selection of Group head}

The transmission takes place through the selection of group head the data processing point on the center of the quad shaped gauze attains the group head position. This done by calculating the mid-point for each quad shaped group and the data processing point on the mid-point is selected to be group head such that each head is located at a distance that can be covered in one flit by the other data processing point, the selection of group head is shown in the Fig 1 collected set of information from each data collection point is sent to

The group head for transmission this is done using a channel access method. The data processing point continuously transmits data and the channel accessing method checks whether the medium where the communication is to take place is free and if it is free the data from the data processing point is sent to the head. On the chance of conflict due to concurrent transfer of information by more than one data processing point the arbiter waits for a predefined time with a minimal pause on transmission and then continues ahead the with process is shown in Fig 2. The conflict detection time could be given as $2 \mathrm{~T}$ where $\mathrm{T}$ is the instant when conflict occurs. This conflict probability between each data point could be eliminated using an expected waiting to its predefined waiting period by setting a space to the retransferring of the information after the conflict during the transmission of information of same preference the expected waiting is given by equation (2)

ISSN: 2582-3167 (online) 
J. Sustain. Wireless Syst.

Vol.01/ No. 02

Pages: 103-115

http://irojournals.com/irosws/

DOI: https://doi.org/10.36548/jsws.2019.2.003

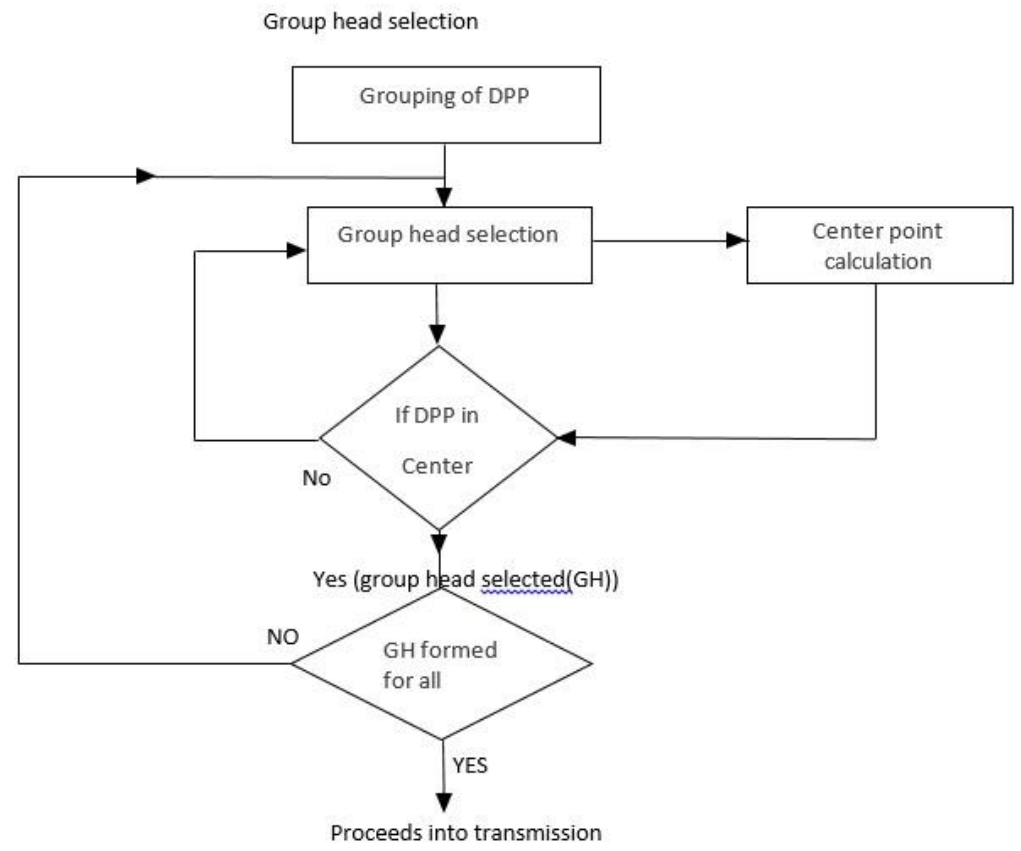

Fig 1.Selection of group head

$$
\text { Ewait }=1 /(n+1) \sum(i=0)^{\wedge} n \text { \#善i } 1=1 /(n+1) n(n+1) / 2=n / 2 \text {, where } n=2 T-1
$$

This expected waiting time Ewait, cancels the delay to be caused in the medium due to waiting for a predefined time.

\section{Pre-eminence Evaluation}

The data now received by each group head using a channel accessing method gets ready to move forward with

ISSN: 2582-3167 (online) 
J. Sustain. Wireless Syst.

Vol.01/ No. 02

Pages: 103-115

http://irojournals.com/irosws/

DOI: https://doi.org/10.36548/jsws.2019.2.003

transmitting it to the base station, for this transmission in order maintain the traffic volume by moving data the across the medium within the given time these information's on each head are to be arranged with preeminence using an optimal scheduler based on dynamic scheduling, the traffic level is calculated based on equation (3) flow chart in Fig 3 shows the optimal scheduler.

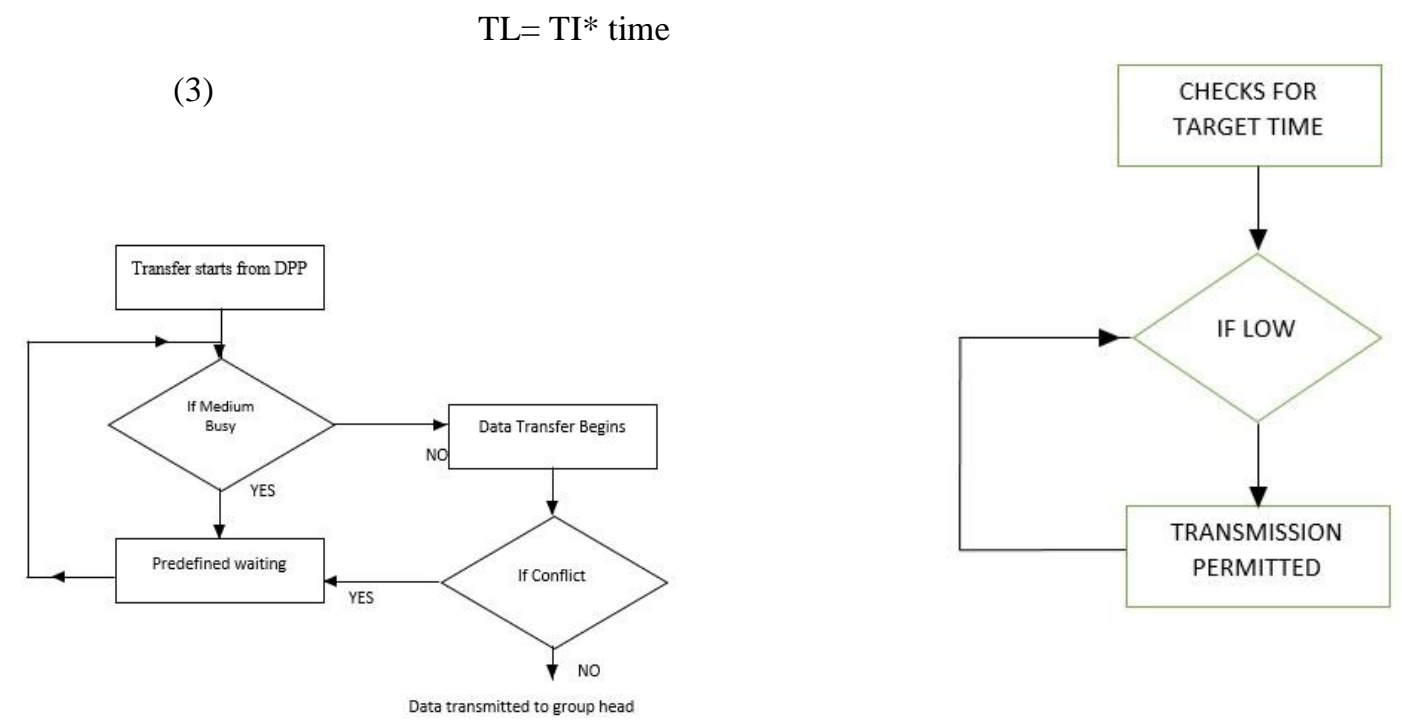

Fig 2 channel access method

Fig 3 optimal scheduler

Each data in the collection is defined with a target time, which is to be met. The preeminence of these data's to be transferred are based on this target time, the one with the shortest duration is set to complete its conveyance first. The preeminence calculation $(\mathrm{Pc})$ is roughly performed using equation (4)

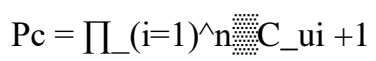

Where, $\mathrm{Cu}$ represents the channel efficiency, the channel efficiency gives us the report of complete task without any loss by equation (5)

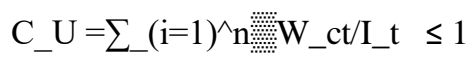


J. Sustain. Wireless Syst.

Vol.01/ No. 02

Pages: 103-115

http://irojournals.com/irosws/

DOI: $\underline{\text { https://doi.org/10.36548/jsws.2019.2.003 }}$

Wct represents the worst case computing time and the It represents the interval of data arrivals. The channel efficiency calculation helps us in knowing whether the information's conveyances within the target time assigned to it without any loss.

\subsection{Improved Energy gleaning by potency segregation}

Improved energy gleaning by potency segregation puts in an offer of ordered usage of energy to turn down the energy use by apprehending energy produced at one time to use it later. This methods is actually based on creating a remodeled accumulator or batteries from its original type. This proceeds by the cleaving of the total cumulated energy along with its gleaned energy available in the accumulator with all the components of a device. The verve segregated between the available components is either used at once it is cumulated or used in an non bulked manner or cumulated for a particular period of time and then utilized in within a aeon of time. The total energy hand out by cleaving is given by equation (6)

$$
\mathrm{CE}=\mathrm{Ri} * \mathrm{Ae}
$$

Where $\mathrm{CE}$ is the total energy hand out, $\mathrm{Ri}$ is the total verve in storage and Ae is the data points or the components in the device. The energy cumulated in the accumulator once cleaved is handed out to each data processing points available in the wireless mesh network. The energy cleaving is done by segregating the gleaned energy into various segments, this segmented energy is further handed out in dues or as a whole to all the data processing points available and the surplus are retrained to be used hereafter, on completely using its energy handed out in dues, the data processing points owes the energy from the surplus retrained. Where the maximum owing is limited by equation (7)

$$
\mathrm{MAXO}=\mathrm{SiRiAe}((\mathrm{CE}-\mathrm{Ci}) / \mathrm{CE})
$$

$\mathrm{Ci}$ is the number of dues that can be received and $\mathrm{Si}$ amount of unused energy, where $\mathrm{Si}$ equal to 1 represents the further availability of dues to be owed and $\mathrm{Si}$ equal to zero represents the complete usage of energy. Once the maximum owing is reached the data processing point is put to a temporary rest and continues once again the temporary rest instant for the data point are is assumed using equation (8). When energy consumed is greater than the total handed out energy at a particular instant. 
J. Sustain. Wireless Syst.

Vol.01/ No. 02

Pages: 103-115

http://irojournals.com/irosws/

DOI: https://doi.org/10.36548/jsws.2019.2.003

$$
\mathrm{EC} \geq \mathrm{CETI}
$$

EC is the energy consume and the TI is the particular instant. The improved energy gleaning enables a supple managing of energy by improved energy gleaning method. The improved energy gleaning method to have a modified storage device without any alteration done physically avoiding a customized space for the modification. It avoids the more area being designated for the improved gleaning energy as the potency segregation is done intellectually and confines the number of resources as well as their maintaining cost. It provides a better stay up time enabling the device to transmit without break. The improved energy gleaning methodology offers a higher quality of utilization of devices as the connection with one enables the connection to the rest. The segregation allows an effective functioning and adaptability. It offers the data processing points of the wireless mesh network to be easily carried into different environment where their energy accumulator is providing a cleaved energy that is been segregated between each data processing point and the energy is devoured only when the information are conveyance to and fro. The conveyancing of data to base station takes place by the channel accessing and preeminence evaluation using an optimal scheduler thus enabling a loss less data transmission device with delay reduction and heightened throughput. Where they are given by delay bounds are given by equation (9)

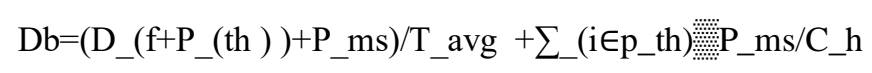

Where Df is the total data frame size, Pth is the path to be enrouted and Pms is maximum affordable size of transmitted information Tavgis the average number of conveyance performed, Chis the channel capacity and $\mathrm{Db}$ is the delay bound. Thus causing a minimum delay in the device. Thus acquiring a vibrant adeptness.

\subsection{Improved Energy Gleaning Supported Routing Stratagem}

The urge to access out a substantial area in networking with solidarity, superfluous communication infrastructure with a cost effective facilities in systems with overloaded conditions and to achieve a higher level of redundancy, providing increased reliability, load balancing and fault tolerance avoiding failure in conveyance, or trail breaks. The routing ability based on group management with improved energy gleaning methodology is involved. The trail requisition from DPP with details about complete size of data to be transmitted and its anticipated span contained in it, the cleaved energy segmented to the trail request enables it to travel along the medium. The mid-way spread nodes, source and destined checks the trail requests contained information. The contained particulars on satisfying the equation (10) and (11) allows the trail formation otherwise dropped. 
J. Sustain. Wireless Syst.

Vol.01/ No. 02

Pages: 103-115

http://irojournals.com/irosws/

DOI: $\underline{\text { https://doi.org/10.36548/jsws.2019.2.003 }}$

$$
\begin{gathered}
\mathrm{ErD}+\mathrm{LD}+\mathrm{EG}=\mathrm{PthETLD}+\mathrm{SDET} \\
\mathrm{ErD}+\mathrm{LD}+\mathrm{EG}=2 \mathrm{PthETLD}+2 \mathrm{SDET}
\end{gathered}
$$

ErD amount of energy segmented through improved energy gleaning for the complete data to transmitted, LD is the life time of the total data to be transmitted, EG it is the energy gained in accumulator by improved energy gleaning, $\mathrm{SD}$ is the size of the total information, ET energy required for transmission, Pth is the path to be enrouted.

Mdr $\geq$ TavgET, Mdr $\geq 2$ Tavg ET

Mdr is the maximum energy used for transmission, Tavgis the average number of conveyance performed, ET energy required for transmission. The DPP once again send an alternative trail requisition with the information in it with changed particulars. On receiving the trail request the destined DPP seeks for more trail requisition with changed particulars and establishes a trail with a maximum values and sends a message in return as trail respond. As the conveyance begins the DPP starts to look for the source address in the routing table of the trail requisition to determine the trail to be taken once detected to be clone the trail requisition is dropped to avoid the formation of the hoop and reduce congestion and finalizes with a trail that is short to be routed. Thus providing a conveyance free of congestion with high reliability, fault tolerance, throughput, lossless and delay management

\section{Result Evaluation}

The result evaluation are done to analyze the performance under the grounds of total transmitted information and delay. The measurement gives the interdependence of the complete information to be transmitted and the throughput achieved on an improved energy gleaning method using potency segregation which would be difficult for the systems using normal accumulators. The proposed method of transmission using the improved gleaning methodologies compared to the previous methods without it and their performance are analyzed. Assumed that the power consumption of each DPP is 5joules, the DPP size is represented in 200 bits, the conveyance energy required is given by 100 joules at the data rate of $5 \mathrm{Mbps}$ and the number of DPP engaged would be 20 to 40 . The proposed is simulated using network simulator to check its performance analysis with the previous existing

\section{(a) Gross Delivery Ratio}

ISSN: 2582-3167 (online) 
J. Sustain. Wireless Syst.

Vol.01/ No. 02

Pages: 103-115

http://irojournals.com/irosws/

DOI: https://doi.org/10.36548/jsws.2019.2.003

It gives the total information that reached the destined compared to the net amount of the transmitted information.

The gross delivery rate can be given as in equation (12)

$$
\text { GDR = net amount of information received /Information transmitted }
$$

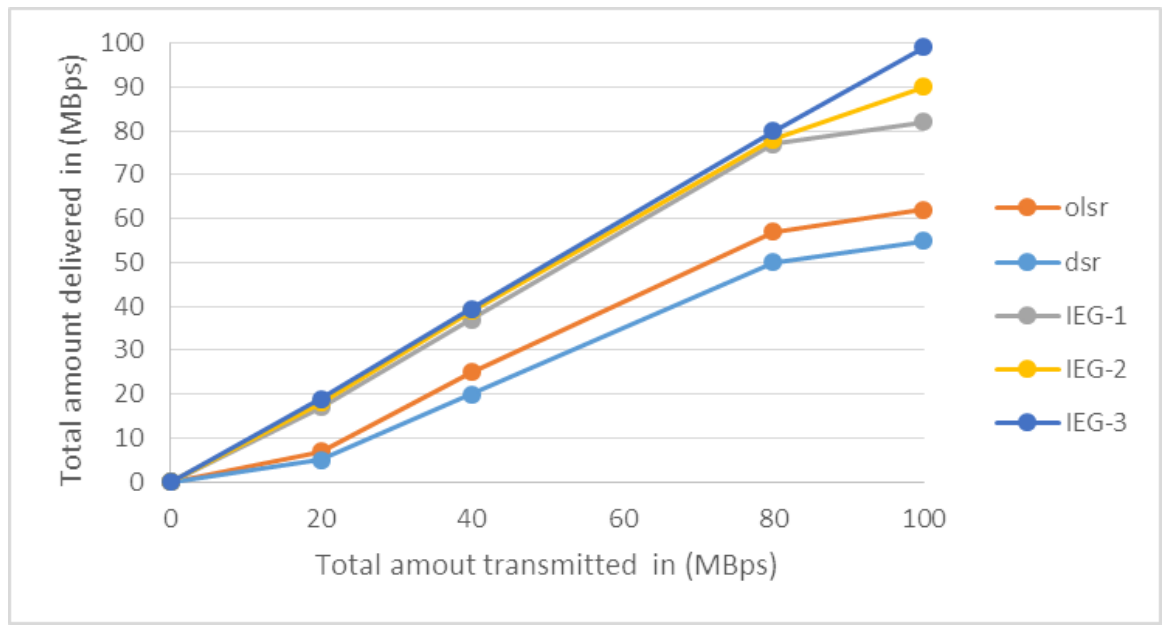

\section{Fig 4 Gross Delivery Ratio}

The graph in Fig 4 shows that the proposed method has an uninterrupted high ratio of delivery because of the improved gleaning of the accumulator when compared to the other methods. It shows the difference in amount of information delivered to the amount of information transmitted. The comparison shows that the proposed with improved energy gleaning method transmits more when compared to the other two that are not engaged with the Improved energy gleaning, due to its low energy capability is to be contented with interrupted transmission and has lost more number of transmitted information than the proposed.

\section{(b) Delay determination}

The delay determination depends on the number of total transmission taken place, the size of the information to be transmitted and the size of the total data frame. It is given as the time taken for the information to reach its destined end subtracted from the time instant at which it was transmitted is given in equation (13) 
J. Sustain. Wireless Syst.

Vol.01/ No. 02

Pages: 103-115

http://irojournals.com/irosws/

DOI: $\underline{\text { https://doi.org/10.36548/jsws.2019.2.003 }}$

Delay determination $=$ time taken to reach destination-time instant of transmission

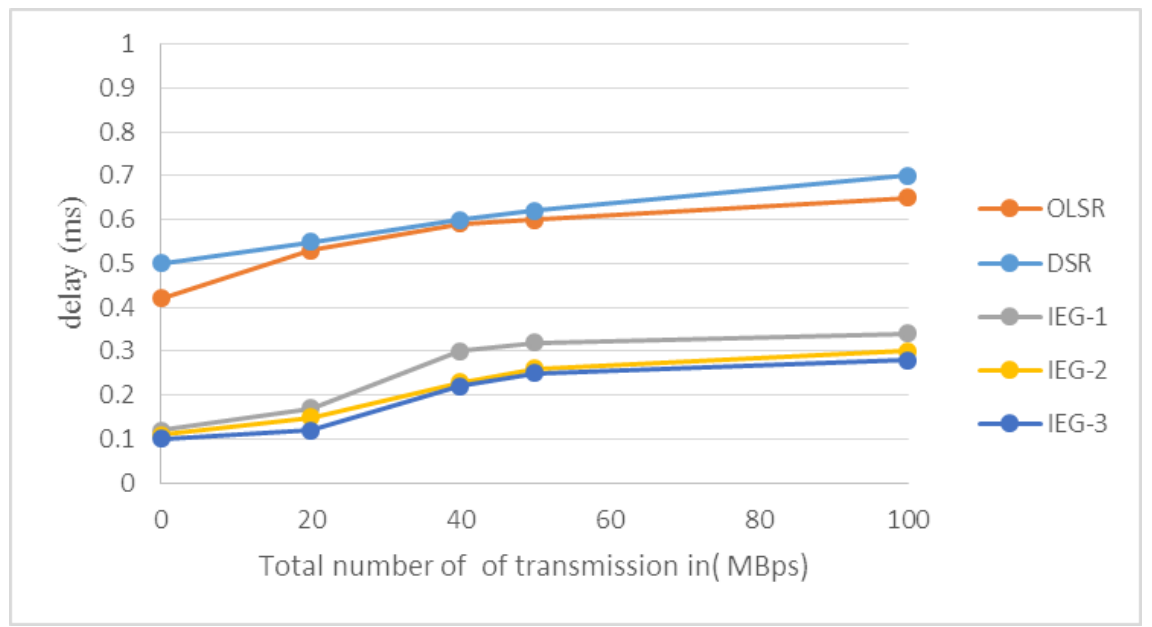

\section{Fig 5 Delay Determination}

The Fig 5 gives the delay obtained during transmission shows that the methods without the improved energy gleaning causes greater delay because of the interrupted transmission due to the failure of effective energy organization. Thus proving the proposed method to be better compared to the previous methods. Thus the performance analysis show that the improved energy gleaning offers better performance.

\section{Conclusion}

The proposed improved energy gleaning using potency segregation on wireless mesh networks offers a better

ISSN: 2582-3167 (online) 
J. Sustain. Wireless Syst.

Vol.01/ No. 02

Pages: 103-115

http://irojournals.com/irosws/

DOI: $\underline{\text { https://doi.org/10.36548/jsws.2019.2.003 }}$

affinity providing maximum adaption. It offers a heightened reliability by having reduced loss on the information transmitted and allows a higher utilization of devices and higher stay up time enabling an interruption clear and a congestion clear conveyancing by proper trail requisition and trial reply caused by the improved energy gleaning methodology, so the wireless mesh networks based on the proposed methodology has an heightened endurance to failures and better managing capability on energy. Which is proved better by the performance check undertaken on the basis of delay and gross delivery ratio on a network simulator and compared with the methods that are not contained with the facility of energy administering and evinced to have an improved quality of service.

\section{References}

[1] Wang, Gang, and Yanyuan Qin. "MAC Protocols for Wireless Mesh Networks with Multi-beam Antennas: A Survey." In Future of Information and Communication Conference, pp. 117-142. Springer, Cham, 2019.

[2] Bashir, Muhammad Nauman, and Kamaludin Mohamad Yusof. "Green Mesh Network of UAVs: A Survey of Energy Efficient Protocols across Physical, Data Link and Network Layers." In 2019 4th MEC International Conference on Big Data and Smart City (ICBDSC), pp. 1-6. IEEE, 2019.

[3] Wang, Jianfeng, Yuguang Fang, and Dapeng Wu. "Enhancing the performance of medium access control for WLANs with multi-beam access point." IEEE Transactions on Wireless Communications 6, no. 2 (2007): 556-565.

[4] Teng, Rui, Huan-Bang Li, Bing Zhang, and Ryu Miura. "Differentiation presentation for sustaining internet access in a disaster-resilient homogeneous wireless infrastructure." IEEE Access 4 (2016): 514-528.

[5] Zhou, Lei, Guixia Kang, Ningbo Zhang, and Jianming Cheng. "Spectral efficiency guaranteed sustainable routing for energy renewable wireless mesh networks." In 2015 International Conference on Wireless Communications \& Signal Processing (WCSP), pp. 1-5. IEEE, 2015.

[6] Puniran, K. N., Robiah Ahmad, and Rudzidatul Akmam Dziyauddin. "RF energy harvesting with multiple sources in wireless sensor network." In 2017 IEEE 4th International Conference on Smart Instrumentation, Measurement and Application (ICSIMA), pp. 1-5. IEEE, 2017.

[7] Attia, Maroua Ben, Kim-Khoa Nguyen, and Mohamed Cheriet. "QoS-aware software-defined routing in smart community network." Computer Networks 147 (2018): 221-235.

[8] Li, Jilong, Bhagya Nathali Silva, Muhammad Diyan, Zhenbo Cao, and Kijun Han. "A clustering based routing algorithm in IoT aware Wireless Mesh Networks." Sustainable cities and society 40 (2018): 657666.

[9] Tu, Wanqing. "Data-Driven QoS and QoE Management in Smart Cities: A Tutorial Study." IEEE Communications Magazine 56, no. 12 (2018): 126-133. 
J. Sustain. Wireless Syst.

Vol.01/ No. 02

Pages: 103-115

http://irojournals.com/irosws/

DOI: https://doi.org/10.36548/jsws.2019.2.003

[10] Singh, Madhusudan. "Wireless Mesh Networks Architecture." In Node-to-Node Approaching in Wireless Mesh Connectivity, pp. 11-14. Springer, Singapore, 2019.

[11]Ertürk, Mehmet Ali, Muhammed Ali Aydin, Luca Vollero, and Roberto Setola. "IEEE 802.11 s Mesh Network Analysis for Post Disaster Communication." In International Telecommunications Conference, pp. 53-59. Springer, Singapore, 2019.

[12] Li, Shu, Jeong Geun Kim, Doohee Han, and Kye San Lee. "A Survey of Energy-Efficient Communication Protocols with QoS Guarantees in Wireless Multimedia Sensor Networks." Sensors 19, no. 1 (2019): 199. 